\title{
Observational aspects of magnetic accretion: Doppler tomography.
}

\author{
Stephen B. Potter ${ }^{1}$ \\ South African Astronomical Observatory \\ Cape Town, South Africa \\ E-mail:sbp@saao.ac.za
}

Enrico J. Kotze

South African Astronomical Observatory and

Astrophysics, Cosmology and Gravity Centre (ACGC), Department of Astronomy, University of Cape

Town,

Cape Town, South Africa

E-mail: ejk@saao.ac.za

\section{Vanessa A. McBride}

South African Astronomical Observatory and Astrophysics, Cosmology and Gravity Centre (ACGC), Department of Astronomy, University of Cape Town,

Cape Town, South Africa

E-mail:vanessa@saao.ac.za

We have applied our inside-out Doppler tomography technique to investigate the emissions from magnetic CVs, specifically the polar V834 Cen. We find that the inside-out projection leads to a redistribution of the relative contrast levels compared to the standard Doppler projection. The various emitting components, such as the ballistic and magnetically confined accretion flows, are more intensified and enhanced: the inside-out projection exposes the low velocity emission details, from the secondary and ballistic stream, that are overly compacted in the standard projection. Similarly, it enhances the high velocity emission details that are washed out in the standard projection, in particular the funnelling of the emission from the magnetically confined accretion flow as it falls towards the primary. Furthermore we have extended the technique to also model the orbitally modulated flux variations. This in turn provides additional information that permits the construction of Doppler tomograms that reveal the amplitude and phase variation from the system's emission components. 
Palermo, Italy

\section{Introduction}

The study of Cataclysmic Variables (CVs) in general not only advances our understanding of this class of object in their own right, but also provides the opportunity to explore a broad range of astrophysical phenomena. The most obvious being accretion. CVs have been pivotal to the development of accretion disc theory. This is because they are nearby (and hence bright), evolve on short time-scales (hours to weeks) and are therefore ideal for micro-arcsecond imaging techniques such as Doppler tomography from which one can probe the binary constituents (the stream, disc, secondary and white dwarf).

Marsh \& Horne (1988) introduced Doppler tomography, a technique initially developed to use orbitally phase-resolved spectra of a CV to construct a two-dimensional tomogram (velocity image) of its accretion disc. Doppler tomography fundamentally changed the interpretation of complex line profiles observed in the phase-resolved spectra of CVs, mCVs and other interacting binary systems (see, e.g., Marsh 2001). Another notable extension to the standard technique is modulation Doppler tomography, introduced by Steeghs (2003). It is a technique which maps emission components which varies harmonically as a function of the orbital period. Three tomograms are constructed simultaneously, i.e., one for the average emission distribution and two for the variable part in terms of its sine and cosine amplitudes. Recent references to the application of this technique mostly involve the spectroscopic studies of X-ray binaries, e.g. Peris et al. (2015) and Čechura, Vrtilek, \& Hadrava (2015).

Kotze, Potter \& McBride (2015, hereafter Paper I) introduced inside-out Doppler tomography, a complementary extension to the standard technique. It reverses the velocity axis to create an inside-out Doppler coordinate frame. Inside-out tomograms are constructed independently of the corresponding standard tomograms by directly projecting phase-resolved spectra onto the inside-out framework. The objective was to explore a more intuitive velocity layout for Doppler tomograms that rectified their inside-out appearance. The results presented in Paper I also showed that the inside-out projection can enhance high velocity emission details which are washed out in the standard projection. Similarly, it can expose low velocity emission details which are overly compacted. We note that our preliminary work on this technique was initially presented at the The Golden Age of Cataclysmic Variables and Related Objects - II, 2013(Kotze \& Potter 2015).

In this paper we apply our inside-out projection technique and a variant form of flux modulation mapping to the Doppler tomography of mCVs. High inclination polars provide the best opportunity to exploit these tomographic techniques. In section 2 we describe our new development of the Doppler tomographic technique and demonstrate its application to obtain new insights from orbitally phase-resolved spectroscopy of the polar V834 Cen (section 3). In section 4 we introduce flux modulated inside-out Doppler tomography and its application to the polar V834 Cen. 

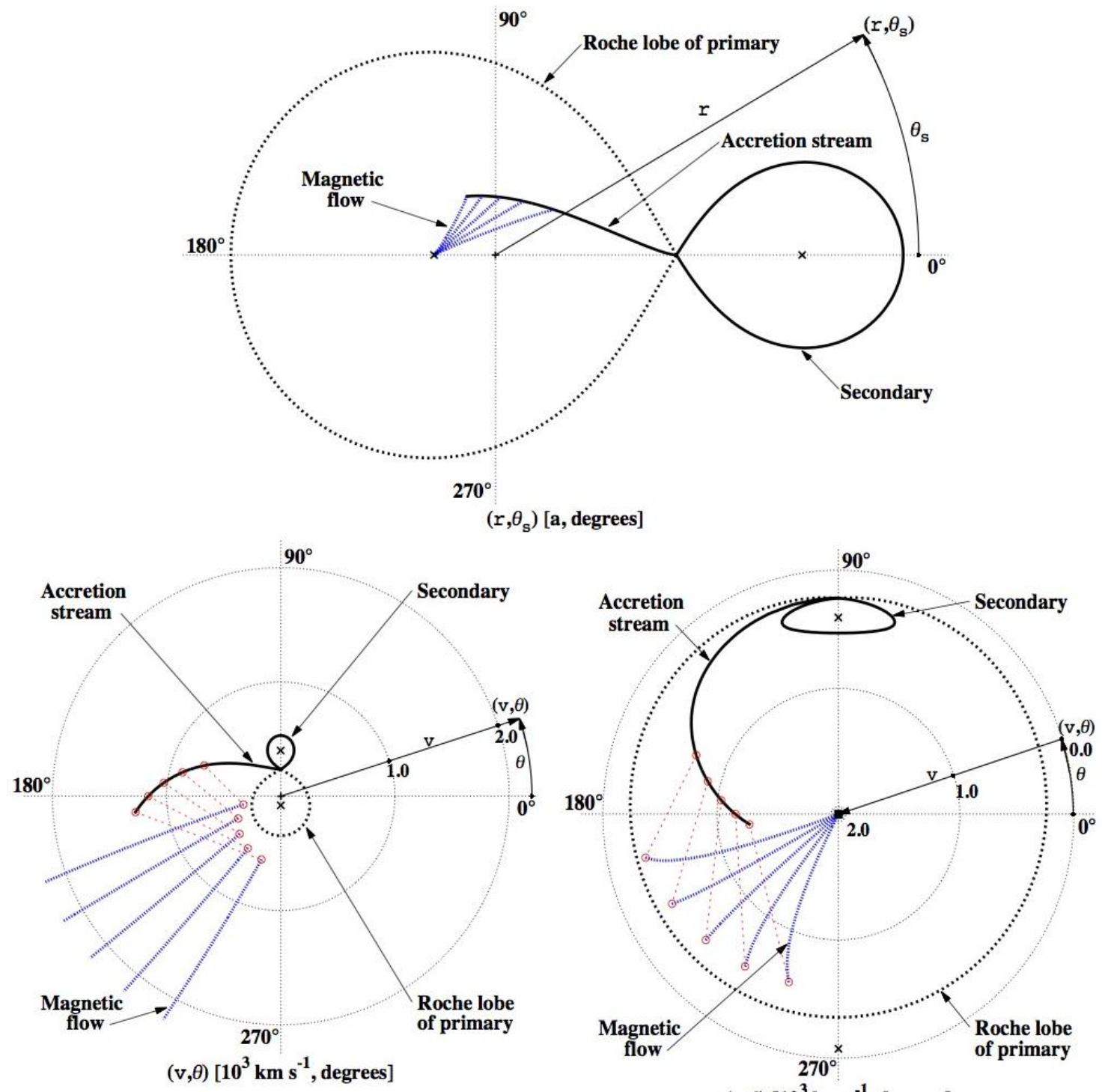

$(\mathrm{v}, \theta)\left[10^{3} \mathrm{~km} \mathrm{~s}^{-1}\right.$, degrees]

Figure 1. Standard spatial and velocity, and inside-out velocity polar coordinates for a model $\mathrm{mCV}$. The top and bottom left panels show the standard spatial and velocity polar coordinate layout, respectively. The bottom right panel shows the inside-out velocity polar coordinate layout. The assumed binary system parameters for the model are: a primary mass of $0.8 \mathrm{M} \odot$; a mass ratio of $\mathrm{q}=$ 0.2 ; an orbital period of $0.083333 \mathrm{~d}(\sim 120 \mathrm{~min})$ and an inclination of $87^{\circ}$. The C.O.M. of the binary is marked with a plus $(+)$ while that of the primary and secondary are marked with crosses $(\times)$. Overlays are shown for the Roche lobe of the primary, the accretion stream from the secondary towards the primary (up to an azimuth angle of $60^{\circ}$ ), as well as for magnetic dipole field lines at $10^{\circ}$ intervals from $20^{\circ}$ to $60^{\circ}$ in azimuth around the primary. The assumed dipole axis azimuth and co-latitude are $35^{\circ}$ and $15^{\circ}$, respectively.

\section{Inside-out Doppler tomography of mCVs}

We first review a polar coordinate frame that co-rotates with the binary system. Paper I asserted that a polar coordinate frame is more conducive to the circularly symmetric nature of Doppler tomograms. It gives a more direct indication of velocities and directions, i.e., it describes velocities in terms of its magnitude and direction which is more intuitive than describing it in terms of its $\mathrm{x}$ - and $\mathrm{y}$-axial components. 
The top panel of Fig. 1 shows the spatial polar coordinates for a model $\mathrm{mCV}$ including the roche lobes of the primary and secondary, the ballistic stream and the magnetically confined accretion along a set of magnetic dipole field lines. The spatial coordinates are the radial distance $r$ from the origin, i.e., the binary's centre of mass (C.O.M.) and the polar angle $\theta \mathrm{s}$ (not to be confused with $\theta$ in the velocity frame) measured in an anti-clockwise direction from the line between the binary's C.O.M. and the secondary. The $r$ coordinate is normalised by the binary separation $a$. The orbital motion is counter-clockwise and the point of mid-eclipse of the primary by the secondary is defined as the binary orbital phase zero.

The bottom left panel of Fig. 1 shows the corresponding velocity map in polar coordinates. The velocity magnitude $v$ increases linearly outwards from the origin and the velocity direction is the polar angle $\theta$ measured anti-clockwise from the line drawn from the origin horizontally to the right (i.e., the $0^{\circ}$-line). For example, the secondary has a velocity magnitude of $\sim 400 \mathrm{~km} \mathrm{~s}^{-1}$ with a velocity direction (angle) of $90^{\circ}$. The increasing velocity of the ballistic accretion stream is reflected in the outwards curve as it accelerates to the left away from the secondary. Similarly, the profiles of the dipole trajectories radiate outwards from lower velocities, where they leave the orbital plane, towards higher velocities as they fall down onto the primary. In this velocity frame they appear to diverge as they fall towards the white dwarf.

The bottom right panel of Fig. 1 shows the inside-out velocity map. In the inside-out velocity polar coordinate frame, zero velocity is on the outer circumference and the maximum velocities are around the centre origin of the coordinate frame. The velocity magnitude $v$ now increases linearly inwards from zero velocity towards the origin, while the velocity direction is still given by the polar angle $\theta$ measured anticlockwise from the $0^{\circ}$-line. The profile of the secondary is now upside down since it is orbiting as a solid body with its outer surface moving faster than its inner (closer to L1) surface. The outer bounded circular ring area, i.e., between zero velocity and the dashed line, is the Roche lobe of the primary. As one can see, in this inside-out framework the profile of the ballistic stream now curves inwards as it accelerates towards higher velocities. In addition, the profiles of the accreting dipole trajectories now converge as they accelerate inwards towards the primary. 


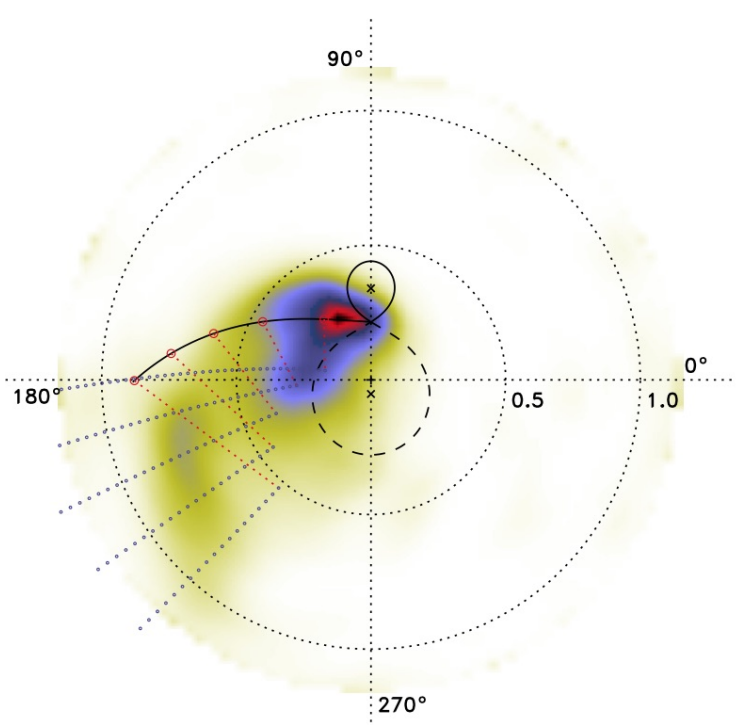

$(\mathrm{v}, \theta)\left[10^{5} \mathrm{~km} \mathrm{~s}^{-1}\right.$, degrees $]$

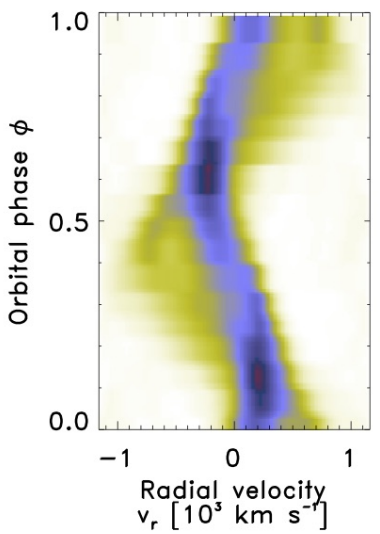

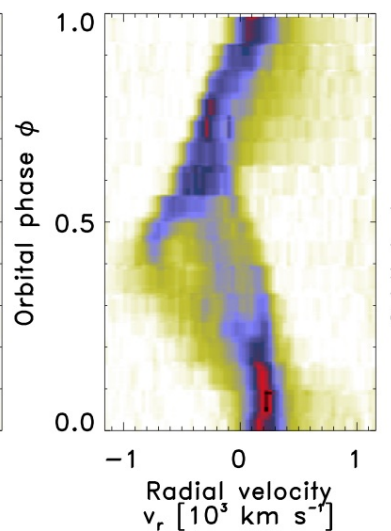

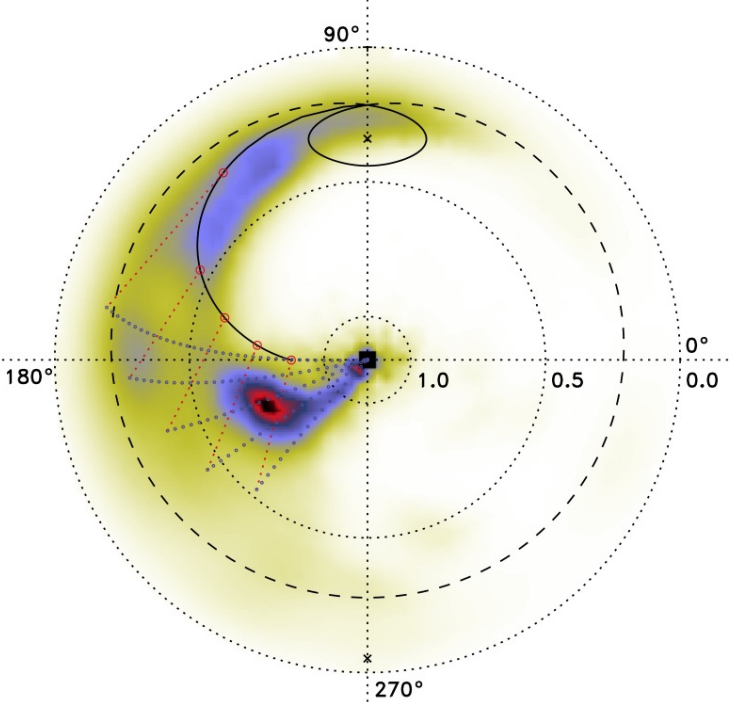

$(v, \theta)\left[10^{3} \mathrm{~km} \mathrm{~s}^{-1}\right.$, degrees $]$

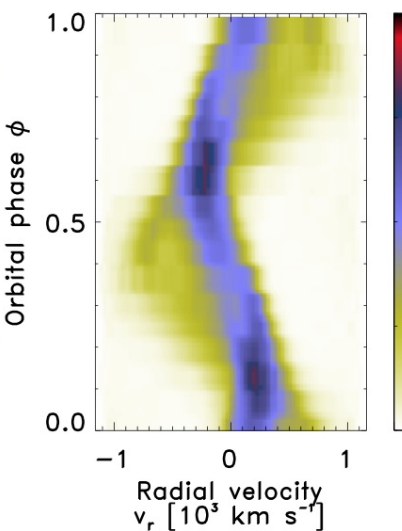

Figure 2. Doppler tomography of V834 Cen. The standard and inside-out tomograms are shown top left and right, respectively. The bottom middle panel shows the input trailed spectra while the bottom left and right panels show the reconstructed trailed spectra for the corresponding tomogram. The model velocity profile overlay shown in both tomograms is based on the model used by Potter et al. (2004) with an inclination angle of $50^{\circ}$, a primary mass of $0.85 \mathrm{M} \odot$, a mass ratio $\mathrm{q}=0.154$ and an orbital period of 0.070497518 $\mathrm{d}(\sim 101.5 \mathrm{~min}$; Schwope et al. 1993). The Roche lobes of the primary (dashed line) and the secondary (solid line) as well as a single particle ballistic trajectory from the L1 point up to $45^{\circ}$ in azimuth around the primary (solid line), are included in the overlay.

Magnetic dipole trajectories are calculated from $5^{\circ}$ to $45^{\circ}$ in azimuth around the primary (thin dotted lines) at $10^{\circ}$ intervals using a dipolar axis azimuth and co-latitude of $\sim 36^{\circ}$ and $\sim 20^{\circ}$ (Potter et al. 2004), respectively. The first of the dipole connections (small circles) are at $\left(360 \mathrm{~km} \mathrm{~s}^{-1}, 135^{\circ}\right)$ and the first dipole trajectory starts at $\left(150 \mathrm{~km} \mathrm{~s}^{-1}, 162^{\circ}\right)$. Consecutive trajectories start at locations with progressively higher velocities and polar angles.

\section{Inside-out Doppler tomography of the polar V834 Cen}

Having laid the frame work for inside-out Doppler tomography in the previous section, we now apply the technique to the polar V834 Cen. V834 Cen is a bright non-eclipsing polar. Potter et al. (2004) presented standard Doppler tomography of V834 Cen that exposed dominant emission from the secondary and (or) from around the inner Lagrangian point (L1). Less discernible emission from the ballistic stream and extended diffuse emission considered to be consistent with the magnetically confined accretion flow. Fig. 2 shows the standard and inside-out Doppler tomography based on the HeII $(\lambda 4686)$ emission line presented by Potter et al. (2004). 
In the standard tomogram the emission from the irradiated secondary is blended with emission from the ballistic stream creating an extremely bright ridge in the velocity range $\left(250-500 \mathrm{~km} \mathrm{~s}-1,90-150^{\circ}\right)$ which dominates the brightness scale of the tomogram. In the inside-out tomogram this blended emission

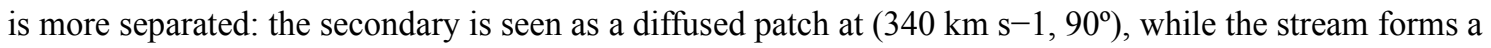
brighter ridge along the model stream trajectory in the velocity range $\left(300-400 \mathrm{~km} \mathrm{~s}-1,115-140^{\circ}\right)$.

The emission associated with the magnetically confined accretion, as it leaves the orbital plane in the threading region, is seen in both tomograms in the velocity range $\left(0-500 \mathrm{~km} \mathrm{~s}-1,170-230^{\circ}\right)$. Also, in both tomograms the emission considered to be consistent with the magnetically confined accretion, as it flows down to the primary, is centred on the ridge of brightness covering the velocity range $(700-1000$ $\mathrm{km} \mathrm{s}-1,185-235^{\circ}$ ). However, in the inside-out tomogram the funnelling of the emission, as it falls towards the primary along the dipole trajectories, is more clearly defined and intensified as it extends to higher velocities. 


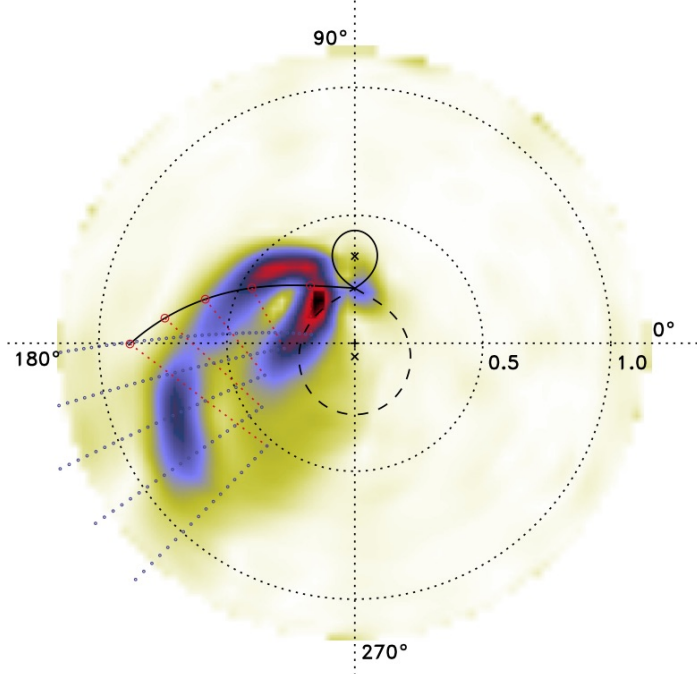

$(\mathrm{v}, \theta)\left[10^{3} \mathrm{~km} \mathrm{~s}^{-1}\right.$, degrees $]$

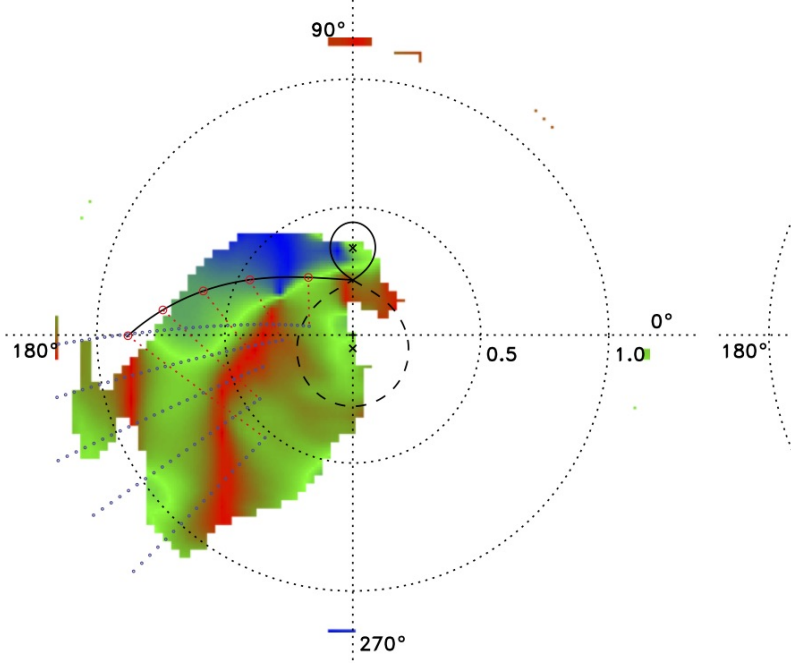

$(v, \theta)\left[10^{3} \mathrm{~km} \mathrm{~s}^{-1}\right.$, degrees $]$

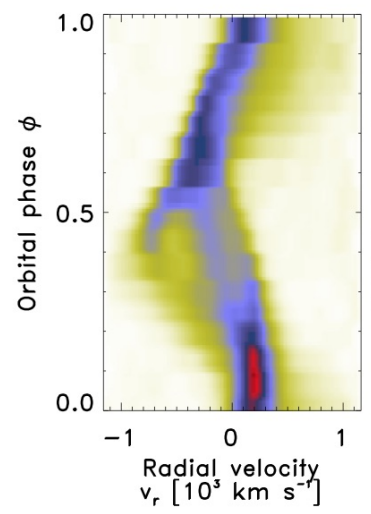

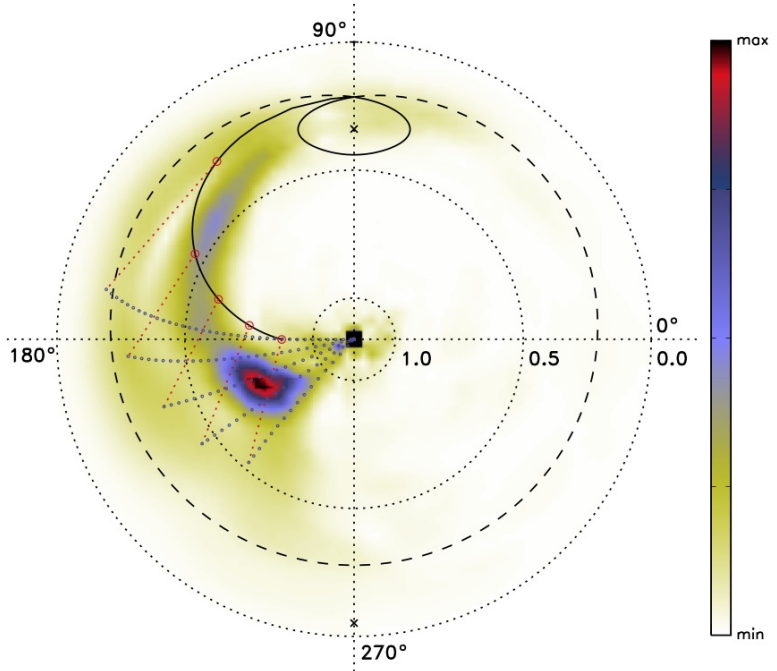

$(\mathrm{v}, \theta)\left[10^{3} \mathrm{~km} \mathrm{~s}^{-1}\right.$, degrees $]$

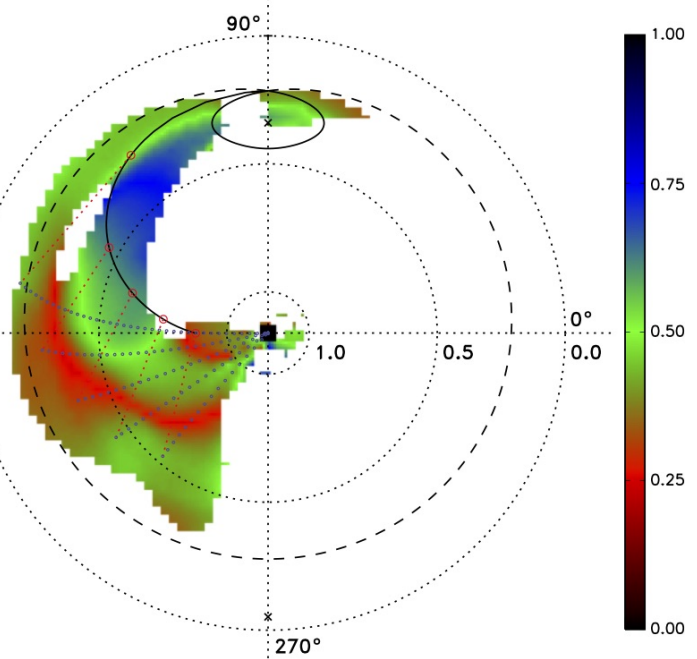

$(\mathrm{v}, \theta)\left[10^{3} \mathrm{~km} \mathrm{~s}^{-1}\right.$, degrees $]$

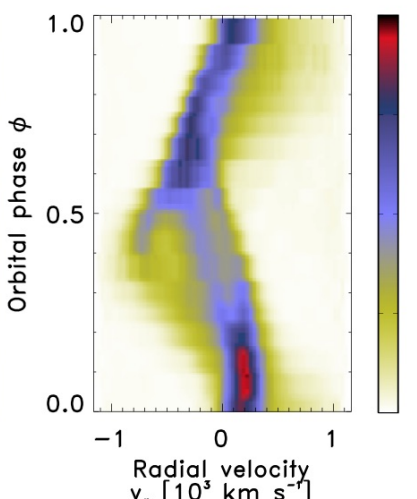

Figure 3. Flux modulation mapping of V834 Cen. The standard and inside-out velocity maps are shown left and right, respectively. The top two panels show the modulation amplitude maps and the middle two panels show the phase of maximum flux maps. The phase of maximum flux map shows only pixels where the corresponding modulation amplitude is at least $7.5 \%$ of the maximum amplitude. The bottom middle panel shows the input trailed spectra while the bottom left and right panels show the summed reconstructed trailed spectra from the corresponding half-phase tomograms. The model velocity profile overlay shown in all the tomograms is the same as the one in Fig. 2. 


\section{Flux modulated Inside-out Doppler tomography of the polar V834 Cen}

One of the axioms of Doppler tomography (Marsh 2001) is that the technique assumes that all points in the binary system are equally visible at all times. This implies that it is possible to construct a tomogram using spectra covering half of a phase only. The fact that this axiom is violated in most CVs and mCVs (especially in the higher inclination systems) actually enables us to selectively eliminate various emission components from the tomogram by using spectra from half of a phase only, thus allowing otherwise less obvious components to become more enhanced (e.g., Potter et al. 2004). In reality the emission/absorption lines are flux modulated at the orbital period which is attributed to the viewing geometry of the system, e.g., a self eclipse or the aspect variation of an emission component. Doppler tomography effectively presents a phase-averaged map of the emission distribution in the system. Phasedependent details, in the orbitally flux modulated spectra, will therefore not be recovered in the reconstructed spectra. Recognising this, Steeghs (2003) introduced modulation Doppler tomography, a technique which maps emission components which modulate sinusoidally as a function of the orbital period. This is achieved through the simultaneous construction of three tomograms, i.e., one for the average emission distribution and two for the variable part in terms of its sine and cosine amplitudes.

Exploiting the principles introduced by Steeghs (2003) and the method used by Potter et al. (2004) we present a variant form of flux modulation mapping. We construct ten tomograms by using spectra from consecutive half-orbits (i.e., phases, 0.0 to $0.5, \ldots, 0.4$ to $0.9,0.5$ to $0.0,0.6$ to $0.1, \ldots, 0.9$ to 0.4 ). We then assume that the flux from an emission component varies harmonically over the observed phases such that one can extract the amplitude (Aj), phase-offset $(\varphi \mathrm{j})$ and average $(\mathrm{Bj})$ of the flux modulation for each of the $j$-th pixels across the ten tomograms. The variation in each pixel across the ten tomograms is fit with a simple sinusoidal of the form

$F i j=A j \sin [2 \pi(\Phi i+\varphi j)]+B j$

Fij and $\Phi \mathrm{i}$ are, respectively, the flux in the j-th pixel and the mid-phase value of the i-th half-phase tomogram. The amplitude for each pixel can be used to produce an amplitude map which helps to identify which emission components are modulated. Adding 0.25 to the fitted phase-offset $(\varphi)$ for each pixel produces a map for the phase of maximum flux. This shows at which phase an emission component appears brightest to an observer.

The standard and inside-out flux modulation mapping results for V834 Cen are shown in Fig. 3 (left and right panels respectively). The lower panels show the input (middle) and reconstructed trailed spectra (left and right panels, same format as Fig. 2). As expected, the reconstructed trailed spectra better resemble the input spectra when compared to the reconstructed spectra of Fig. 2. In particular the obvious brightening in flux between phases 0.0-0.2. In addition, the multiple components between phases 0.2-0.5 are now better reproduced as too is the flux level on the high blue velocity component at phase $0.4-0.5$.

Having now accurately reproduced the trailed spectra, we can derive the amplitude (Aj), phase-offset ( $\varphi \mathrm{j})$ and average $(\mathrm{Bj})$ of the flux modulation doppler tomograms. The average $(\mathrm{Bj})$ is effectively the normal tomogram (not shown) and appears identical to those of the top panels in Fig. 2. The amplitude (Aj) tomograms are displayed in the top panels of Fig. 3, for both the standard and inside-out maps. They clearly intensify and better resolve the ballistic and the magnetically confined accretion flows. The secondary is also flux modulated, but since V834 Cen is a mid-inclination non-eclipsing system the amplitude of the modulation is not so dominant.

The middle panels of Fig. 3 map the phase-offset $(\varphi j)$ at which maximum flux occurs. The map has been colour coded to represent phase. So, for example, it is expected that the irradiated face of the secondary appears brightest at phase 0.5 , which corresponds to the green colour coding. In this case the inside-out 
tomogram is easier to interpret. Starting at the L1 point and following the model ballistic stream as a guide, one can see that the mapped ballistic stream starts green and progresses through to blue. The implication being that the part of the ballistic stream closest to the secondary is seen brightest at phase 0.5 i.e., when its inner irradiated surface in pointing most in our direction. Later parts of the ballistic stream appear at their brightest centred on phase $\sim 0.75$ (blue). The map also shows a second low velocity component running parallel to the ballistic stream which also shows maximum brightness at phase 0.5 (green). This would be consistent with the irradiated inner surface of the magnetically confined accretion curtain, the later parts of which appear to show maximum brightness at earlier phases $\sim 0.25$ (red). This is consistent with an irradiated magnetically confined accretion curtain being most face-on at these phases.

\section{Summary}

We have applied our inside-out Doppler tomography technique to investigate the emissions from the polar V834 Cen. The inside-out projection leads to a redistribution in the relative contrast levels in and amongst the emitting components such as the ballistic and magnetically confined accretion flows. The inside-out projection better exposes low velocity emission details, such as the secondary and ballistic stream, that are overly compacted in the standard projection. Similarly, it enhances the high velocity emission details that are washed out in the standard projection. In particular the funnelling of the emission from the magnetically confined accretion flow as it falls towards the primary. Furthermore we have extended the technique to also model the orbitally modulated flux variations. This in turn provides additional information that permits the construction of Doppler tomograms that reveal the amplitude and phase information from the system's emission components.

We next plan to apply the technique to a range of mCVs with different properties, such as magnetic field strength, inclination, mass ratio, orbital period and accretion states etc. mCVs also include the intermediate polars where spin tomograms may be constructed in order to investigate the magnetically confined accretion flows locked in the spinning white dwarf reference frame.

Our code, with examples, can be downloaded from http://www.saao.ac.za/ ejk/doptomog/main.html

\section{References}

[1] Marsh T. R., Horne K., Images of accretion discs. II - Doppler tomography, 1988, MNRAS, 235, 269

[2] Marsh T. R., Doppler Tomography, 2001, LNP, 573, 1

[3] Steeghs D., Extending emission-line Doppler tomography: mapping-modulated line flux, 2003, MNRAS, 344, 448

[4] Peris C. S., et al. Tomography of X-ray Nova Muscae 1991: evidence for ongoing mass transfer and stream-disc overflow, 2015, MNRAS, 449, 1584

[5] Čechura J., Vrtilek S. D., \& Hadrava P., Interpreting the X-ray state transitions of Cygnus X-1, 2015, MNRAS, 450, 2410

[6] Kotze E. J., Potter S. B., \& McBride V. A., Exploring inside-out Doppler tomography: nonmagnetic cataclysmic variables, 2015, A\&A, 579, 77

[7] Kotze E. J., Potter S. B., New Insights from Inside-Out Doppler Tomography, 2015, AcPPP, 2, 170 